\title{
Palladium-Catalyzed Intramolecular Selenocarbamoylation of Alkynes with Carbamoselenoates: Formation of $\alpha$-Alkylidene- $\beta$-Lactam Framework
}

\author{
Masashi Toyofuku, ${ }^{\dagger}$ Shin-ichi Fujiwara, ${ }^{*}{ }^{\ddagger}$ Tsutomu Shin-ike, ${ }^{\ddagger}$ Hitoshi Kuniyasu, ${ }^{\dagger}$ Nobuaki Kambe ${ }^{* \dagger}$ \\ ${ }^{\dagger}$ Department of Molecular Chemistry, Graduate School of Engineering, Osaka University, \\ Suita, Osaka 565-0871, Japan \\ Department of Chemistry, Osaka Dental University, \\ Hirakata, Osaka 573-1121, Japan \\ e-mail: fujiwara@cc.osaka-dent.ac.jp, kambe@chem.eng.osaka-u.ac.jp
}

\section{Supporting Information}

1. General Comments

2. Preparation of Carbamoyl Chlorides

3. Procedures and Characterization of Reaction Materials

4. Procedures and Characterization of Reaction Products

5. References and Notes 


\section{Genaral Comments}

THF was distilled from benzophenone ketyl just prior to use. 1-Octyne, toluene, and $\mathrm{CH}_{2} \mathrm{Cl}_{2}$ were distilled from $\mathrm{CaH}_{2}$. $\mathrm{Pd}\left(\mathrm{PPh}_{3}\right)_{4}$ was prepared according to the literature procedure. ${ }^{\mathrm{S} 1}$ Carbamoyl chlorides except $N, N$-dimethylcarbamoyl chloride were synthesized from triphosgene and $N$-alkyl- $N$-alk-2-ynylamines.

Melting points were determined on a Yanagimoto Micro Melting Point apparatus. ${ }^{1} \mathrm{H}$ and ${ }^{13} \mathrm{C}$ NMR spectra were recorded on a JEOL JNM-ALICE-400 (400 MHz and $100 \mathrm{MHz}$, respectively) spectrometer using $\mathrm{Me}_{4} \mathrm{Si}$ (in $\mathrm{CDCl}_{3}$ ) as an internal standard. IR spectra were determined on a Perkin Elmer Model 1600 spectrometer. Preparative TLC was conducted by using Wakogel B-5F silica gel (325 mesh). Mass spectra (EI) were taken on a SHIMAZU GCMS-QP2000 operating in the electron impact mode $(70 \mathrm{eV})$ equipped with CBP1-M25-025 column. High-resolution mass spectra (HRMS) were obtained on a JEOL JMS-DX303 in the Instrumental Analysis Center of the Faculty of Engineering, Osaka University. Elemental analyses were performed on Perkin Elmer 240C apparatus.

\section{Prparation of Carbamoyl Chlorides}

$N$-Butyl- $N$-prop-2-ynylcarbamoyl chloride: Typical Procedure. ${ }^{\mathrm{S} 2}$ Into a $200-\mathrm{mL}$ flask were placed triphosgene $(8.3 \mathrm{mmol})$ and $\mathrm{CH}_{2} \mathrm{Cl}_{2}(8 \mathrm{~mL})$ under $\mathrm{N}_{2}$. After cooling to $-78^{\circ} \mathrm{C}$, pyridine $(2.5$ mmol) was added. To the solution was then added slowly $\mathrm{N}$-butyl- $\mathrm{N}$-prop-2-ynylamine (25 mmol), prepared from prop-2-ynyl chloride and $n$-butylamine, in $\mathrm{CH}_{2} \mathrm{Cl}_{2}(4 \mathrm{~mL})$ at the same temperature. After the mixture was warmed up to room temperature, the stirring was continued for 2 days until gas-emission ceased. The expected carbamoyl chloride was obtained in $66 \%$ yield by distillation $\left(4 \mathrm{mmHg}, 80^{\circ} \mathrm{C}\right)$ as colorless oil contaminated with a small amount of pyridine hydrochloride and was used without further purification. 
CAUSION: Triphosgene decomposes slightly to generate highly-poisonous phosgene in air. All operation should be carried out in a well-ventilated hood.

\section{Procedures and Characterization of Reaction Materials}

\section{$S e$-Phenyl $N, N$-dimethylcarbamoselenoate (1): Typical procedure.}<smiles>CN(C)C(=O)[Se]c1ccccc1</smiles>

Into a 100-mL flask were placed elemental selenium (16 mmol) and THF (20 mL) under Ar and the suspension was cooled to $0{ }^{\circ} \mathrm{C}$. $\mathrm{PhLi}\left(0.94 \mathrm{M}\right.$ in $\mathrm{Et}_{2} \mathrm{O}$-cyclohexane, $\left.16 \mathrm{~mL}, 15 \mathrm{mmol}\right)$ was added slowly to prepare PhSeLi and the stirring was continued for 5 min. To the pale yellow solution of PhSeLi was then added $N, N$-dimethylcarbamoyl chloride $(16 \mathrm{mmol})$ in $10 \mathrm{~mL}$ of THF at the same temperature, and the mixture was warmed up to room temperature and stirred overnight. After the mixture was poured into brine $(50 \mathrm{~mL})$ and extracted with $\mathrm{Et}_{2} \mathrm{O}(30 \mathrm{~mL} \mathrm{X} \mathrm{4})$, the combined organic phase was dried with $\mathrm{MgSO}_{4}$. The solvents were removed in vacuo and the residue was purified by silica gel column chromatography ( $n$-hexane/Et $\left.{ }_{2} \mathrm{O}=1 / 1\right)$ to afford Se-phenyl $N, N$-dimethylcarbamoselenoate (1) in $73 \%$ yield: white solid; mp $34{ }^{\circ} \mathrm{C} ;{ }^{1} \mathrm{H}$ NMR $(400$ $\left.\mathrm{MHz}, \mathrm{CDCl}_{3}\right) \delta 2.98(\mathrm{~s}, 6 \mathrm{H}), 7.33-7.58(\mathrm{~m}, 5 \mathrm{H}) ;{ }^{13} \mathrm{C} \mathrm{NMR}\left(100 \mathrm{MHz}, \mathrm{CDCl}_{3}\right) \delta 36.7,37.1,126.4$ $\left({ }^{1} J_{S e-C}=91.7 \mathrm{~Hz}\right), 128.4,128.6,136.2\left({ }^{2} J_{S e-C}=10.6 \mathrm{~Hz}\right), 163.9 ; \operatorname{IR}(\mathrm{KBr}) 2925,1678(\mathrm{C}=\mathrm{O}), 1477$, 1361, 1255, 1094, 891, 746, 692, 624, $480 \mathrm{~cm}^{-1}$; MS(EI), m/e (relative intensity, \%) $229\left(\mathrm{M}^{+}, 5\right)$, 157(6), 77(5), 72(100). Anal. Calcd for $\mathrm{C}_{9} \mathrm{H}_{11}$ NOSe: $\mathrm{C}, 47.38 ; \mathrm{H}, 4.86 ; \mathrm{N}, 6.14$. Found: $\mathrm{C}, 47.27$; H, 4.76; N, 6.20 . 


\section{Se-Phenyl $N$-methyl- $N$-prop-2-ynyl carbamoselenoate (3a).}<smiles>C#CCN(C)C(=O)[Se]c1ccccc1</smiles>

Purified by silica gel column chromatography ( $n$-hexane/Et $\left.{ }_{2} \mathrm{O}=1 / 1\right) ; 44 \%$ yield as a $s$-cis/trans mixture; colorless oil; ${ }^{1} \mathrm{H}$ NMR (400 MHz, $\mathrm{CDCl}_{3}$ ) $\delta 2.27$ (brs, $1 \mathrm{H}$ ), 3.09 (brs, $3 \mathrm{H}$ ), 4.22 (brs, 2 $\mathrm{H})$, 7.33-7.60 (m, $5 \mathrm{H}) ;{ }^{13} \mathrm{C} \mathrm{NMR}\left(100 \mathrm{MHz}, \mathrm{CDCl}_{3}\right) \delta 34.6$ (major, minor), 37.7 (major), 40.0 (minor), 72.5 (major), 73.3 (minor), 77.0 (major), 77.5 (minor), 126.0, 128.8, 128.9, $136.2\left({ }^{2} J_{S e-C}\right.$ = 9.7 Hz), 164.6(br); IR(NaCl) 3292, 3056, $1674(\mathrm{C}=\mathrm{O}), 1438,1373,1256,1192,1067,1022$, 740, $690 \mathrm{~cm}^{-1}$; MS(EI), m/e (relative intensity, \%) 253 (M+1 4), 157(10), 96(100), 77(9), 55(15). Anal. Calcd for $\mathrm{C}_{11} \mathrm{H}_{11} \mathrm{NOSe}$ : C, 52.39; H, 4.40; N, 5.55. Found: C, 52.10; H, 4.22; N, 5.61.

\section{$S e$-Phenyl $N$-butyl- $N$-prop-2-ynylcarbamoselenoate (3b).}

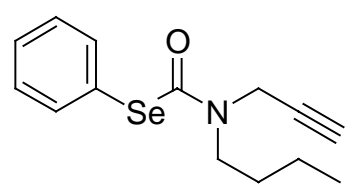

Purified by silica gel column chromatography ( $n$-hexane/Et $\left.{ }_{2} \mathrm{O}=1 / 1\right) ; 59 \%$ yield as a $s$-cis/trans mixture; colorless oil; ${ }^{1} \mathrm{H}$ NMR (400 MHz, $\left.\mathrm{CDCl}_{3}\right) \delta 0.99$ (br, $\left.3 \mathrm{H}\right), 1.40$ (br, $\left.2 \mathrm{H}\right), 1.70$ (br, $2 \mathrm{H}$ ), 2.25 (br, $1 \mathrm{H}), 3.44$ (br, $2 \mathrm{H}), 4.21$ (br, $2 \mathrm{H}), 7.32-7.60$ (m, $5 \mathrm{H}) ;{ }^{13} \mathrm{C} \mathrm{NMR}\left(100 \mathrm{MHz}, \mathrm{CDCl}_{3}\right) \delta$ 13.5 (major, minor), 20.0 (major, minor), 29.6 (minor), 29.9 (major), 35.8 (major), 38.2 (minor), 48.0 (major, minor), 72.2 (major), 73.0 (minor), 77.0 (major), 78.0 (minor), 126.1, 128.8, 128.9, $136.3\left({ }^{2} J_{S e-C}=10.0 \mathrm{~Hz}\right), 164 ; \mathrm{IR}(\mathrm{NaCl}) 3293,2959,1674(\mathrm{C}=\mathrm{O}), 1439,1393,1303,1199,1105$, 1022, 740, $690 \mathrm{~cm}^{-1}$; MS(EI), m/e (relative intensity, \%) $295\left(\mathrm{M}^{+}, 2\right), 157$ (13), 138 (100), 82 (16), 77(8), 57(57). Anal. Calcd for $\mathrm{C}_{14} \mathrm{H}_{17}$ NOSe: $\mathrm{C}, 57.10 ; \mathrm{H}, 5.92 ; \mathrm{N}, 4.92$. Found: C, 57.15; H, 5.82; $\mathrm{N}, 4.76$. 
$S e$-Phenyl $N$-benzyl- $N$-prop-2-ynylcarbamoselenoate (3c).<smiles>C#CCN(Cc1ccccc1)C(=O)[Se]c1ccccc1</smiles>

Purified by silica gel column chromatography ( $n$-hexane/Et $2 \mathrm{O}=1 / 1) ; 29 \%$ yield as a $s$-cis/trans mixture; colorless oil; ${ }^{1} \mathrm{H}$ NMR (400 MHz, $\left.\mathrm{CDCl}_{3}\right)$ $\delta 2.27-2.37$ (m, $\left.1 \mathrm{H}\right)$, 4.02-4.15 (m, $\left.2 \mathrm{H}\right), 4.70$ (brs, $2 \mathrm{H}$ ), 7.32-7.61 (m, $10 \mathrm{H}) ;{ }^{13} \mathrm{C} \mathrm{NMR}\left(100 \mathrm{MHz}, \mathrm{CDCl}_{3}\right.$ ) $\delta 35.2$ (major), 37.0 (minor), 49.9 (minor), 51.1 (major), 72.6 (major), 73.3 (minor), 77.1 (major), 77.5 (minor), 126.0, 127.4 (br), 127.7 (br), 128.5 (br), 128.7, 128.8, 134.6 (br), $136.3\left({ }^{2} J_{S e-C}=10.1 \mathrm{~Hz}\right), 164.5 ; \operatorname{IR}(\mathrm{NaCl}) 3290$, 3061, $1667(\mathrm{C}=\mathrm{O}), 1496,1455,1393,1184,1076,959,740 \mathrm{~cm}^{-1} ; \mathrm{MS}(\mathrm{EI}), \mathrm{m} / \mathrm{e}$ (relative intensity, \%) 329 ( $\left.\mathrm{M}^{+}, 1\right), 172$ (23), 157 (3), 91 (100), 77 (3). Anal. Calcd for $\mathrm{C}_{17} \mathrm{H}_{15} \mathrm{NOSe}$ C, 62.20; H, 4.61; N, 4.27. Found: C, 62.43; H, 4.57; N, 4.26.

$S e$-Phenyl $N$-benzyl- $N$-pent-2-ynylcarbamoselenoate (3d).<smiles>CCC#CCN(Cc1ccccc1)C(=O)[Se]c1ccccc1</smiles>

Purified by silica gel column chromatography ( $n$-hexane/Et $2 \mathrm{O}=4 / 1) ; 54 \%$ yield as a $s$-cis/trans mixture; pale yellow oil; ${ }^{1} \mathrm{H}$ NMR (400 MHz, $\left.\mathrm{CDCl}_{3}\right) \delta$ 1.14-1.27 (m, $\left.3 \mathrm{H}\right), 2.21$ (brs, $2 \mathrm{H}$ ), 4.01-4.14 (m, $2 \mathrm{H}), 4.69$ (s, $2 \mathrm{H})$ 7.34-7.65(m, $10 \mathrm{H}) ;{ }^{13} \mathrm{C} \mathrm{NMR}\left(100 \mathrm{MHz}, \mathrm{CDCl}_{3}\right) \delta 12.5$ (major, minor), 13.9 (major, minor), 35.9 (major), 37.8 (minor), 50.0 (minor), 51.1 (major), 72.7 (minor), 73.1 (major), 86.6 (major), 87.3 (minor), 126.4, 127.4 (br), 127.7 (br), 127.7 (br), 128.4, 128.7, 
$134.0(\mathrm{br}) 136.4\left({ }^{2} J_{S e-C}=10.6 \mathrm{~Hz}\right), 164.4 ; \mathrm{IR}(\mathrm{NaCl}) 2976,1673(\mathrm{C}=\mathrm{O}), 1438,1392,1173,982$, 740, $690 \mathrm{~cm}^{-1}$; MS(EI), m/e (relative intensity, \%) 357 ( $\mathrm{M}^{+}, 0.2$ ), 200 (22), 157 (9), 143 (4), 91 (100), 77 (3). Anal. Calcd for $\mathrm{C}_{19} \mathrm{H}_{19}$ NOSe: C, 64.04; H, 5.37; N, 3.93. Found: C, 63.76; H, 5.40; N, 3.97.

$S e$-Phenyl $N$-methyl- $N$-[3-(p-chlorophenyl)prop-2-ynyl]carbamoselenoate (3e).

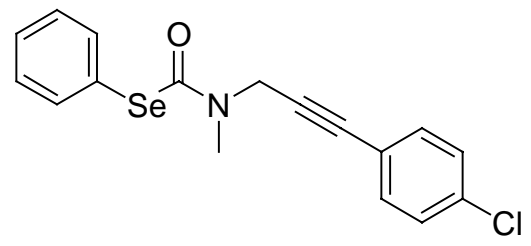

Purified by silica gel column chromatography ( $n$-hexane/Et $\left.{ }_{2} \mathrm{O}=5 / 1\right) ; 48 \%$ yield as a $s$-cis/trans mixture; pale yellow solid; mp $85{ }^{\circ} \mathrm{C} ;{ }^{1} \mathrm{H}$ NMR $\left(400 \mathrm{MHz}, \mathrm{CDCl}_{3}\right) \delta 3.15$ (s, $\left.3 \mathrm{H}\right), 4.44$ (brs, $2 \mathrm{H}$ ), 7.30-7.62(m, $9 \mathrm{H}) ;{ }^{13} \mathrm{C}$ NMR (100 MHz, $\mathrm{CDCl}_{3}$ at $\left.23{ }^{\circ} \mathrm{C}\right) \delta 34.6$ (major, minor), 38.5 (major), 41.2 (minor), 83.3 (major, minor), 84.1 (major, minor), 120.7 (br), 126.3, 128.5, 128.8, 128.9, 132.8, 134.4 (br), 136.4, 164.7 (br); ${ }^{13} \mathrm{C}$ NMR (100 MHz, $\mathrm{CDCl}_{3}$ at $-40{ }^{\circ} \mathrm{C}$ ); 34.6 (major), 34.8 (minor), 38.2 (major), 40.7 (minor), 82.6 (major), 82.9 (minor), 83.5 (minor), 83.7 (major), 119.9 (minor), 120.2 (major), 125.6 (major, minor), 128.2 (major, minor), 128.8 (major, minor), 132.6 (major, minor), 133.9 (major), 134.1 (minor), 136.3 (major), 136.3 (minor), 164.3 (major), 165.0 (minor); IR(KBr) 3070, 2931, $1671(\mathrm{C}=\mathrm{O}), 1488,1376,1263,1089,831,752,688 \mathrm{~cm}^{-1}$; MS(EI), m/e (relative intensity, \%) $363\left(\mathrm{M}^{+}, 1\right), 157(3), 151$ (31), 149 (100), 114 (5), 77(2). Anal. Calcd for $\mathrm{C}_{17} \mathrm{H}_{14}$ ClNOSe: C, 56.29; H, 3.89; N, 3.86. Found: C, 56.02; H, 3.83; N, 3.82. 


\section{Se-Phenyl $N$-butyl- $N$-pent-4-ynylcarbamoselenoate (8a).}<smiles>C#CCCCN(CCCC)C(=O)[Se]c1ccccc1</smiles>

Purified by silica gel column chromatography ( $n$-hexane/ $\left.\mathrm{Et}_{2} \mathrm{O}=3 / 1\right) ; 38 \%$ yield as a s-cis/trans mixture; colorless oil; ${ }^{1} \mathrm{H}$ NMR (400 MHz, $\left.\mathrm{CDCl}_{3}\right) \delta$ 0.90-1.00 (m, $\left.3 \mathrm{H}\right), 1.30-2.05(\mathrm{~m}, 9 \mathrm{H})$, 3.29-3.45 (m, $4 \mathrm{H})$, 7.25-7.60 (m, $\left.5 \mathrm{H}) ;{ }^{13} \mathrm{C} \mathrm{NMR} \mathrm{(100} \mathrm{MHz,} \mathrm{CDCl}_{3}\right) \delta 13.7$ (major, minor), 16.0 (major, minor), 20.0 (major, minor), 26.4 (major), 27.4 (minor), 29.8 (minor), 30.8 (major), 47.1 (major), 47.7 (minor), 48.0 (minor), 49.2 (major), 68.8 (major), 69.4 (minor), 82.4 (minor), 83.4 (major), 126.4, 128.4, 128.6, $136.2\left({ }^{2} J_{S e-C}=9.7 \mathrm{~Hz}\right), 163.4 ; \mathrm{IR}(\mathrm{NaCl}) 3296,2958,1668(\mathrm{C}=\mathrm{O})$, 1438, 1402, 1200, 1155, 1108, 942, 740, $690 \mathrm{~cm}^{-1}$; MS(EI), m/e (relative intensity, \%) 323( $\mathrm{M}^{+}$, 0.2), 166 (100), 157 (9), 82 (8), 77(6). Anal. Calcd for $\mathrm{C}_{16} \mathrm{H}_{21}$ NOSe: C, 59.62; H, 6.57; N, 4.35. Found: C, 59.24; H, 6.28; N, 4.41 .

\section{Se-Phenyl $N$-butyl- $N$-hex-5-ynylcarbamoselenoate (8b).}

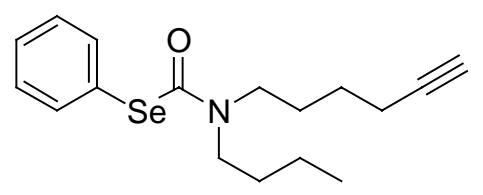

Purified by silica gel column chromatography ( $n$-hexane/Et $\left.{ }_{2} \mathrm{O}=3 / 1\right) ; 39 \%$ yield as a $s$-cis/trans mixture; colorless oil; ${ }^{1} \mathrm{H}$ NMR (400 MHz, $\left.\mathrm{CDCl}_{3}\right) \delta$ 0.90-1.00 (m, $\left.3 \mathrm{H}\right), 1.28-1.99(\mathrm{~m}, 9 \mathrm{H})$, 2.19-2.28 (m, $2 \mathrm{H}), 3.28-3.37$ (m, $4 \mathrm{H})$, 7.25-7.60 (m, $5 \mathrm{H}) ;{ }^{13} \mathrm{C} \mathrm{NMR}\left(100 \mathrm{MHz}, \mathrm{CDCl}_{3}\right) \delta 13.9$ (major, minor), 18.2 (major, minor), 20.2 (major, minor), 25.7 (major, minor), 26.9 (major), 27.9 (minor), 29.9 (minor), 31.0 (major), 47.6 (major), 47.9 (minor), 48.6 (minor), 48.9 (major), 68.6 (major), 68.9 (minor), 83.5 (minor), 83.9 (major), 126.6, 128.5, 128.8, $136.4\left({ }^{2} J_{S e-C}=9.7 \mathrm{~Hz}\right.$ ), 163.4; IR(NaCl) 3296, 2956, $1673(\mathrm{C}=\mathrm{O}), 1438,1402,1199,1149,1107,740,690,631 \mathrm{~cm}^{-1}$; 
MS(EI), m/e (relative intensity, \%) 357( $\left.\mathrm{M}^{+}, 0.6\right), 180$ (100), 157 (10), 81 (71), 79 (22). Anal.

Calcd for $\mathrm{C}_{17} \mathrm{H}_{23} \mathrm{NOSe}$ C, 60.71; H, 6.89; N, 4.16. Found: C, 60.48; H, 6.81; N, 4.36.

$S e$-Phenyl $N$-pent-4-ynyl- $N$-prop-2-ynylcarbamoselenoate (10).

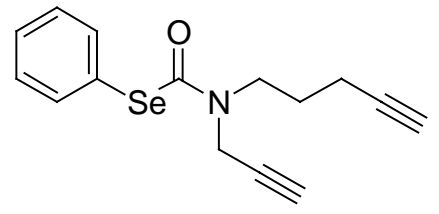

Purified by silica gel column chromatography ( $n$-hexane/Et $\left.{ }_{2} \mathrm{O}=3 / 1\right) ; 59 \%$ yield as a $s$-cis/trans mixture; colorless oil; ${ }^{1} \mathrm{H}$ NMR (400 MHz, $\left.\mathrm{CDCl}_{3}\right) \delta$ 1.80-2.33 (m, $\left.6 \mathrm{H}\right), 3.58(\mathrm{t}, J=7.4 \mathrm{~Hz}, 2 \mathrm{H})$, 4.23 (brs, $2 \mathrm{H}$ ), 7.33-7.61 (m, $5 \mathrm{H}) ;{ }^{13} \mathrm{C}$ NMR (100 MHz, $\mathrm{CDCl}_{3}$ ) $\delta 15.9$ (major, minor), 26.4 (minor), 26.9 (major), 36.2 (major), 38.6 (minor), 47.3 (major, minor), 68.9 (minor), 69.5 (major), 72.5 (major), 73.2 (minor), 77.5 (minor), 77.8 (major), 82.4 (major), 82.9 (minor), $125.9\left({ }^{1} J_{S e-C}=\right.$ $93.2 \mathrm{~Hz}), 128.7$ (two peaks overlapped), $136.3\left({ }^{2} J_{S e-C}=10.6 \mathrm{~Hz}\right), 164.2 ; \operatorname{IR}(\mathrm{NaCl}) 3295,2937$, $1668(\mathrm{C}=\mathrm{O}), 1440,1394,1259,1186,1153,1065,741,690 \mathrm{~cm}^{-1} ; \mathrm{MS}(\mathrm{EI}), \mathrm{m} / \mathrm{e}$ (relative intensity, \%) 305( $\left.\mathrm{M}^{+}, 1\right), 157$ (11), 148 (100), 120(14), 77(10), 68(18). Anal. Calcd for $\mathrm{C}_{15} \mathrm{H}_{15} \mathrm{NOSe}$ C, 59.22; H, 4.97; N, 4.60. Found: C, 58.95; H, 4.88; N, 4.47.

\section{$S$-Phenyl $N$-methyl- $N$-prop-2-ynylcarbamothiolate (12).}

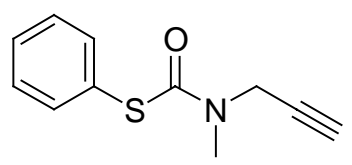

Into a 50-mL flask equipped with a dropping funnel were placed $N$-methyl $N$-prop-2-ynylcarbamoyl chloride $(4.3 \mathrm{mmol})$ and THF $(15 \mathrm{~mL})$ under Ar. A solution of benzenethiol $(4.7 \mathrm{mmol})$ and pyridine $(21.5 \mathrm{mmol})$ in $5 \mathrm{~mL}$ of THF was added dropwise at $0^{\circ} \mathrm{C}$.

The mixture was then warmed up to room temperature and stirred overnight. After white 
precipitates were filtered off, $\mathrm{Et}_{2} \mathrm{O}$ was added to the filtrate and washed with brine, and dried with $\mathrm{MgSO}_{4}$. Evaporation of the solvent and purification by silica gel column chromatography $\left(n\right.$-hexane/ $\left.\mathrm{Et}_{2} \mathrm{O}=3 / 1\right)$ afforded 12 in $66 \%$ yield: ${ }^{1} \mathrm{H}$ NMR $\left(400 \mathrm{MHz}, \mathrm{CDCl}_{3}\right) \delta 2.28($ brs, $1 \mathrm{H})$, 3.12 (brs, $3 \mathrm{H}), 4.21$ (brs, $2 \mathrm{H})$ 7.37-7.50 (m, $5 \mathrm{H}) ;{ }^{13} \mathrm{C} \mathrm{NMR}\left(100 \mathrm{MHz}, \mathrm{CDCl}_{3}\right) \delta 34.1,37.8,72.5$, 77.5, 128.8, 128.5, 128.9, 135.3, 166.6(br); IR(NaCl) 3292, 3060, 2918, $1666(\mathrm{C}=\mathrm{O}), 1476,1441$, 1375, 1262, 1199, 1077, 750, $689 \mathrm{~cm}^{-1}$; MS(EI), m/e (relative intensity, \%) $205\left(\mathrm{M}^{+}, 12\right), 109$ (17), 96 (100). Anal. Calcd for $\mathrm{C}_{11} \mathrm{H}_{11}$ NOS: C, 64.36; H, 5.40; N, 6.82. Found: C, 64.07; H, 5.41; N, 6.72 .

Se-Phenyl 2,2-dimethylpent-4-ynoylselenoate (14).

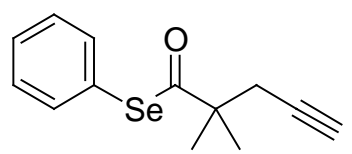

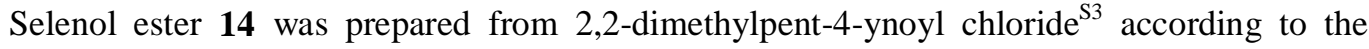
typical procedure of carbamoselenoates and was purified by silica gel column chromatography $(n$-hexane/Et $2 \mathrm{O}=10 / 1) ; 34 \%$ yield; colorless oil; ${ }^{1} \mathrm{H}$ NMR $\left(400 \mathrm{MHz}, \mathrm{CDCl}_{3}\right) \delta 1.39(\mathrm{~s}, 6 \mathrm{H})$, $2.06(\mathrm{t}, J=2.7 \mathrm{~Hz}, 1 \mathrm{H}), 2.50(\mathrm{~d}, J=2.7 \mathrm{~Hz}, 2 \mathrm{H}), 7.34-7.49(\mathrm{~m}, 5 \mathrm{H}) ;{ }^{13} \mathrm{C} \mathrm{NMR}(100 \mathrm{MHz}$, $\left.\mathrm{CDCl}_{3}\right) \delta 24.3,29,3,52.2,71.0,79.9,125.5,128.4,128.8,135.9\left({ }^{2} J_{S e-C}=9.2 \mathrm{~Hz}\right), 205.8$; $\operatorname{IR}(\mathrm{NaCl})$ 3295, 2971, $1714(\mathrm{C}=\mathrm{O}), 1465,1366,911,739,690 \mathrm{~cm}^{-1} ; \mathrm{MS}(\mathrm{EI}), \mathrm{m} / \mathrm{e}$ (relative intensity, \%) $266\left(\mathrm{M}^{+}, 4\right), 157$ (10), 109 (52), 81 (100). Anal. Calcd for $\mathrm{C}_{13} \mathrm{H}_{14} \mathrm{OSe}$ : C, 58.88; H, 5.32. Found: C, 58.87; H, 5.32. 


\section{Procedures and Characterization of Reaction Products}

(Z)-N,N-Dimethy-3-(phenylseleno)non-2-enamide

\section{Selenocarbamoylation}

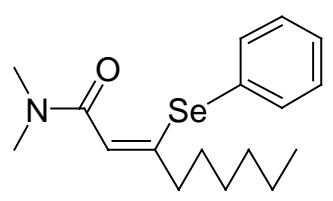

Into a 3-mL flask equipped with a reflux condenser were placed carbamoselenoate $\mathbf{1}(0.5 \mathrm{mmol})$, toluene $(0.3 \mathrm{~mL}), 1$-octyne $(1.5 \mathrm{mmol})$, and $\mathrm{Pd}\left(\mathrm{PPh}_{3}\right)_{4}(0.025 \mathrm{mmol})$ at room temperature under Ar and the solution turned immediately red. After the mixture was refluxed for $14 \mathrm{~h}$, excess 1-octyne and toluene were removed in vacuo. The crude product was separated by preparative TLC ( $n$-hexane/Et $2 \mathrm{O}=5 / 1)$ to give (Z)- $N, N$-dimethy-3-(phenylseleno)non-2-enamide (2) in 40\% yield: brown oil; ${ }^{1} \mathrm{H}$ NMR (400 MHz, $\left.\mathrm{CDCl}_{3}\right) \delta 0.78(\mathrm{t}, J=7.3 \mathrm{~Hz}, 3 \mathrm{H}), 1.02-1.37(\mathrm{~m}, 8 \mathrm{H}), 2.19$ (t, $J=7.3 \mathrm{~Hz}, 2 \mathrm{H}), 3.02(\mathrm{~s}, 3 \mathrm{H}), 3.07(\mathrm{~s}, 3 \mathrm{H}), 6.48(\mathrm{~s}, 1 \mathrm{H}), 7.27-7.38(\mathrm{~m}, 3 \mathrm{H})$, 7.66-7.68 (m, 2 $\mathrm{H}) ;{ }^{13} \mathrm{C}$ NMR $\left(100 \mathrm{MHz}, \mathrm{CDCl}_{3}\right) \delta 14.0,22.3,28.4,29.9,31.2,35.4,37.4,37.9,112.9,128.2$, 128.4, 128.9, $137.0\left({ }^{2} J_{S e-C}=9.7 \mathrm{~Hz}\right), 158.5,166.9$; NOE experiment: irradiation of the vinyl singlet at $\delta 6.48$ resulted in $13 \%$ and $6 \%$ enhancements of the signals at $\delta 3.02-3.07\left(\mathrm{NMe}_{2}\right)$ and $\delta 2.19$ (C-3 methylene triplet), respectively; $\operatorname{IR}(\mathrm{NaCl}) 2928,2857,1622(\mathrm{C}=\mathrm{O}), 1574,1395,1316$, 1262, 1161, 818, 743, 696. $\mathrm{cm}^{-1}$; MS(EI), m/e (relative intensity, \%) $339\left(\mathrm{M}^{+}, 10\right), 295(10), 182$ (100), 157 (4), 72(36). Anal. Calcd for $\mathrm{C}_{17} \mathrm{H}_{25} \mathrm{NOSe}$ C, 60.35; H, 7.45; N, 4.14. Found: C, 60.27; H, 7.37; N, 4.10. 


\section{Selenocarbamoylation}

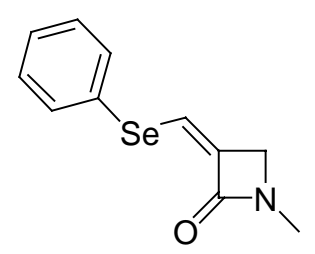

Into a 3-mL flask equipped with a reflux condenser were placed carbamoselenoate 3a $(0.4$ mmol), toluene $(0.5 \mathrm{~mL})$, and $\mathrm{Pd}\left(\mathrm{PPh}_{3}\right)_{4}(0.020 \mathrm{mmol})$ at room temperature under $\mathrm{Ar}$ and the solution turned immediately red. After the mixture was refluxed for $1 \mathrm{~h}$, toluene was removed in vacuo. The crude product was purified by preparative recycling HPLC (eluted with $\mathrm{CHCl}_{3}$ ) to provide (Z)-1-methyl-3-phenylselenomethyleneazetidin-2-one (7a) in 74\% yield: white solid; mp $93{ }^{\circ} \mathrm{C} ;{ }^{1} \mathrm{H}$ NMR $\left(400 \mathrm{MHz}, \mathrm{CDCl}_{3}\right) \delta 2.97(\mathrm{~s}, 3 \mathrm{H}), 3.72(\mathrm{~s}, 2 \mathrm{H}), 6.70(\mathrm{~s}, 1 \mathrm{H}), 7.32-7.57(\mathrm{~m}, 5 \mathrm{H})$;

${ }^{13} \mathrm{C}$ NMR $\left(100 \mathrm{MHz}, \mathrm{CDCl}_{3}\right) \delta 28.9,50.8,119.6\left({ }^{1} J_{S e-C}=121.7 \mathrm{~Hz}\right), 127.7,129.2,130.1,132.6$ $\left({ }^{2} J_{S e-C}=10.6 \mathrm{~Hz}\right), 135.3,163.0$; NOE experiment: irradiation of the allylic proton singlet at $\delta 3.72$ resulted in $2.9 \%$ enhancement of the signal at $\delta 6.70$ (vinyl singlet); $\mathrm{IR}(\mathrm{KBr}) 3045,2901,1728$ $(\mathrm{C}=\mathrm{O}), 1578,1476,1437,1416,1386,1257,1242,1194,1035,902,816,741,690,464 \mathrm{~cm}^{-1}$; MS(EI), m/e (relative intensity, \%) 253 (M+100), 251 (49), 250 (19), 249 (19), 195 (49), 157 (31), 115 (65), 77(25). Anal. Calcd for $\mathrm{C}_{11} \mathrm{H}_{11}$ NOSe: $\mathrm{C}, 52.39 ; \mathrm{H}, 4.40 ; \mathrm{N}, 5.55$. Found: $\mathrm{C}, 52.31 ; \mathrm{H}$, $4.28 ; \mathrm{N}, 5.56$. 


\section{1-Butyl-3-phenylselenomethyleneazetidin-2-one (7b).}

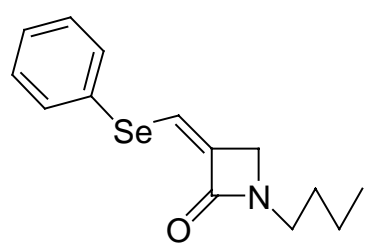

Into a 3-mL flask equipped with a reflux condenser were placed carbamoselenoate $\mathbf{3 b}(0.4$ mmol), toluene $(0.5 \mathrm{~mL})$, and $\mathrm{Pd}\left(\mathrm{PPh}_{3}\right)_{4}(0.020 \mathrm{mmol})$ at room temperature under Ar and the solution turned immediately red. The mixture was refluxed for $1 \mathrm{~h}$ and toluene was removed in vacuo. After the NMR yield and the $E / Z$ ratio were determined by ${ }^{1} \mathrm{H} N M R(85 \%, E / Z=2 / 98)$, the crude product was purified by preparative recycling $\mathrm{HPLC}$ (eluted with $\mathrm{CHCl}_{3}$ ) to provide 1- $n$-butyl-3-phenylselenomethyleneazetidin-2-one (7b) in $60 \%$ yield. An attempt for isolation of isomers failed, so the following spectra and analytical data were obtained from the $E / Z$ mixture: brown oil; ${ }^{1} \mathrm{H}$ NMR $\left(400 \mathrm{MHz}, \mathrm{CDCl}_{3}\right) \delta 0.93(\mathrm{t}, J=7.3 \mathrm{~Hz}, 3 \mathrm{H}), 1.31-1.40$ (tq, $J=7.3,7.1 \mathrm{~Hz}$, $2 \mathrm{H}), 1.50-1.58(\mathrm{dt}, J=7.1,7.3 \mathrm{~Hz}, 2 \mathrm{H}), 3.27(\mathrm{t}, J=7.3 \mathrm{~Hz}, E(2 \mathrm{H})), 3.32(\mathrm{t}, J=7.3 \mathrm{~Hz}, Z(2 \mathrm{H}))$, $3.49(\mathrm{~s}, E(2 \mathrm{H})) 3.71(\mathrm{~s}, Z(2 \mathrm{H})), 6.69(\mathrm{~s}, Z(1 \mathrm{H})), 7.19(\mathrm{~s}, E(1 \mathrm{H})), 7.29-7.31(\mathrm{~m}, 3 \mathrm{H})$, 7.54-7.57 (m, $2 \mathrm{H}) ;{ }^{13} \mathrm{C}$ NMR $\left(100 \mathrm{MHz}, \mathrm{CDCl}_{3}\right) \delta 13.7(E), 13.8(Z), 20.2(E), 20.3(Z), 29.8(Z), 29.9(E)$, $41.6(E), 41.7(Z), 48.3(Z), 48.9(E), 118.6,119.6\left({ }^{1} J_{S e-C}=121.2 \mathrm{~Hz}(Z)\right), 127.7,128.2,129.2$, 129.3, 130.2, $132.6 \mathrm{~Hz}\left({ }^{2} J_{S e-C}=11.1 \mathrm{~Hz}(Z)\right), 133.4,134.8,162.7$ (Z); NOE experiment: Irradiation of the allylic proton singlet at $\delta 3.71$ resulted in $2.1 \%$ enhancement of the signal at $\delta 6.69$ (vinyl singlet); IR(NaCl) 2956, $1739(\mathrm{C}=\mathrm{O}), 1670,1477,1393,1072,740 \mathrm{~cm}^{-1}$; MS(EI), m/e (relative intensity, \%) $295\left(\mathrm{M}^{+}, 100\right), 293$ (49), 252 (34), 223 (37), 195 (57), 157 (31), 115 (70), 77(30). Anal. Calcd for $\mathrm{C}_{14} \mathrm{H}_{17} \mathrm{NOSe}$ C, 57.15; H, 5.82; N, 4.76. Found: C, 56.98; H, 5.76; N, 5.56 . 
(Z)-1-Benzyl-3-phenylselenomethyleneazetidin-2-one (7c).

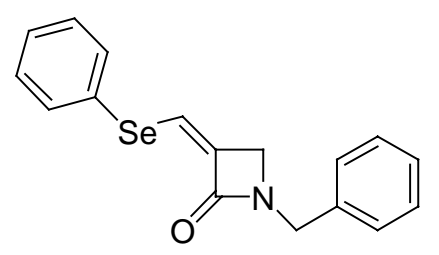

Into a 3-mL flask equipped with a reflux condenser were placed carbamoselenoate $3 \mathbf{c}(0.4$ mmol), toluene $(2 \mathrm{~mL})$, and $\mathrm{Pd}\left(\mathrm{PPh}_{3}\right)_{4}(0.020 \mathrm{mmol})$ at room temperature under $\mathrm{Ar}$ and the solution turned immediately red. After the mixture was refluxed for $1 \mathrm{~h}$, toluene was removed in vacuo. After the NMR yield and the $E / Z$ ratio were determined by ${ }^{1} \mathrm{H} N M R(84 \%, E / Z=3 / 97)$, the crude product was purified by preparative recycling HPLC (eluted with $\mathrm{CHCl}_{3}$ ) to provide 1-benzyl-3-phenylselanylmethylene azetidin-2-one (7c) in 59\% yield. Pure (Z)-7c was obtained by recrystalization from $n$-hexane $/ \mathrm{CHCl}_{3}=1 / 1$ : white solid; mp $88{ }^{\circ} \mathrm{C} ;{ }^{1} \mathrm{H} \mathrm{NMR}(400 \mathrm{MHz}$, $\left.\mathrm{CDCl}_{3}\right) \delta 3.62(\mathrm{~s}, 2 \mathrm{H}), 4.51(\mathrm{~s}, 2 \mathrm{H}), 6.73(\mathrm{~s}, 1 \mathrm{H}), 7.25-7.37(\mathrm{~m}, 8 \mathrm{H}), 7.55-7.58(\mathrm{~m}, 2 \mathrm{H}) ;{ }^{13} \mathrm{C}$ NMR $\left(100 \mathrm{MHz}, \mathrm{CDCl}_{3}\right) \delta 46.1,48.4,120.6\left({ }^{1} J_{S e-C}=122.6 \mathrm{~Hz}\right), 127.4,127.6,127.8,128.4,129.0$, 129.9, $132.6\left({ }^{2} J_{S e-C}=11.1 \mathrm{~Hz}\right), 134.5,135.1,162.6$; NOE experiment: Irradiation of the allyl-proton singlet at $\delta 3.62$ resulted in $4.5 \%$ and $3.1 \%$ enhancements of the signals at $\delta 4.51$ (benzyl singlet) and $\delta 6.73$ (vinyl triplet), respectively; $\operatorname{IR}(\mathrm{KBr}) 3060,1720(\mathrm{C}=\mathrm{O}), 1576,1398$, 1345, 1111, 1073, 822, 743, $700 \mathrm{~cm}^{-1}$; MS(EI), m/e (relative intensity, \%) 329 ( $\left.\mathbf{M}^{+}, 46\right), 195$ (26), 157 (8), 115 (30), 91 (100). Anal. Calcd for $\mathrm{C}_{17} \mathrm{H}_{15}$ NOSe: C, 62.20; H, 4.61; N, 4.27. Found: C, $61.88 ; \mathrm{H}, 4.40 ; \mathrm{N}, 4.28$. 
(Z)-1-Benzyl-3-(1-phenylseleno)propylideneazetidin-2-one (7d).

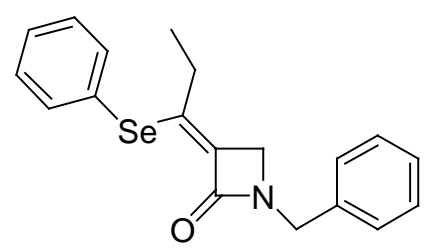

Into a 3-mL flask equipped with a reflux condenser were placed carbamoselenoate $\mathbf{3 d}(0.4$ mmol), toluene $(0.5 \mathrm{~mL})$, and $\mathrm{Pd}\left(\mathrm{PPh}_{3}\right)_{4}(0.020 \mathrm{mmol})$ at room temperature under Ar and the solution turned immediately red. After the mixture was refluxed for $1 \mathrm{~h}$, toluene was removed in vacuo. After the NMR yield and the $E / Z$ ratio were determined by ${ }^{1} \mathrm{H} N M R(93 \%, E / Z=3 / 97)$, the crude product was purified by preparative recycling $\mathrm{HPLC}$ (eluted with $\mathrm{CHCl}_{3}$ ) to provide 1-benzyl-3-(1-phenylseleno)propylideneazetidin-2-one (7d) in $88 \%$ yield. Pure (Z)-7d was obtained by recrystalization from $n$-hexane/ $\mathrm{CHCl}_{3}=1 / 1$ : white solid; mp $102{ }^{\circ} \mathrm{C} ;{ }^{1} \mathrm{H}$ NMR $(400$ $\left.\mathrm{MHz}, \mathrm{CDCl}_{3}\right) \delta 0.93(\mathrm{t}, J=7.3,3 \mathrm{H}), 2.07(\mathrm{q}, J=7.3,2 \mathrm{H}), 3.66(\mathrm{~s}, 2 \mathrm{H}), 4.52(\mathrm{~s}, 2 \mathrm{H}), 7.27-7.37$ (m, $8 \mathrm{H})$, 7.61-7.64 (m, $2 \mathrm{H}) ;{ }^{13} \mathrm{C} \mathrm{NMR}\left(100 \mathrm{MHz}, \mathrm{CDCl}_{3}\right) \delta$ 13.3, 27.8, 46.0, 47.8, 126.9, 127.3, 127.9, 128.0, 128.4, 128.7, 133.0, 135.1, 135.4, $135.5\left({ }^{2} J_{S e-C}=10.6 \mathrm{~Hz}\right), 162.6$; NOE experiment: Irradiation of the allylic proton singlet at $\delta 3.66$ resulted in $2.2 \%$ and $1.6 \%$ enhancements of the signals at $\delta 2.07$ (methylene quartet) and $\delta 4.52$ (benzyl singlet), respectively; $\operatorname{IR}(\mathrm{KBr}) 3058$, 2969, $1730(\mathrm{C}=\mathrm{O}), 1678,1456,1393,1275,1118,1072,962,855,749,704,638 \mathrm{~cm}^{-1}$; MS(EI), m/e (relative intensity, \%) $357\left(\mathrm{M}^{+}, 17\right), 200$ (26), 157 (4), 91 (100). Anal. Calcd for $\mathrm{C}_{19} \mathrm{H}_{19} \mathrm{NOSe}$ : C, 64.04; H, 5.37; N, 3.93. Found: C, 63.93; H, 5.34; N, 3.95. 
(Z)-1-Methyl-3-[(p-chlorophenyl)phenylselenomethylene]azetidin-2-one (7e).

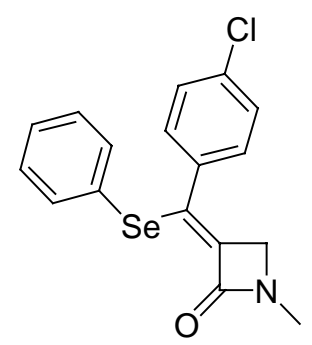

Into a 3-mL flask equipped with a reflux condenser were placed carbamoselenoate $3 \mathbf{e}(0.4$ mmol), toluene $(4 \mathrm{~mL})$, and $\mathrm{Pd}\left(\mathrm{PPh}_{3}\right)_{4}(0.020 \mathrm{mmol})$ at room temperature under Ar and the solution turned immediately red. After the mixture was refluxed for $20 \mathrm{~h}$, toluene was removed in vacuo. The crude product was purified by preparative recycling HPLC (eluted with $\mathrm{CHCl}_{3}$ ) to provide 1-methyl-3-[( $p$-chlorophenyl)phenylselenomethylene $]$ azetidin-2-one (7e) in 76\% yield. After the $E / Z$ ratio was determined by ${ }^{1} \mathrm{H} \operatorname{NMR}(E / Z=11 / 89)$, pure $(Z)-7 e$ was obtained by recrystalization from $n$-hexane/CHCl${ }_{3}=1 / 1$ : pale yellow solid; mp $128{ }^{\circ} \mathrm{C} ;{ }^{1} \mathrm{H} \mathrm{NMR}(400 \mathrm{MHz}$, $\left.\mathrm{CDCl}_{3}\right) \delta 3.01(\mathrm{~s}, 3 \mathrm{H}), 3.91(\mathrm{~s}, 2 \mathrm{H}), 7.06-7.32(\mathrm{~m}, 9 \mathrm{H}) ;{ }^{13} \mathrm{C} \mathrm{NMR}\left(100 \mathrm{MHz}, \mathrm{CDCl}_{3}\right) \delta 28.9$, $51.1,127.4,128.2,128.3,128.6,129.7,129.9,133.9\left({ }^{2} J_{S e-C}=11.1 \mathrm{~Hz}\right), 134.1,135.0,137.7,162.7$; IR (KBr) 3084, 2948, 2874, $1746(\mathrm{C}=\mathrm{O}), 1675,1577,1381,1260,1120,1081,750,704 \mathrm{~cm}^{-1}$; MS(EI), m/e (relative intensity, \%) 363 (M+1 16), 225 (11), 163 (12), 157 (2) 149 (100). Anal. Calcd for $\mathrm{C}_{17} \mathrm{H}_{14}$ CINOSe: C, 56.29; H, 3.89; N, 3.86. Found: C, 56.26; H, 3.75; N, 3.86. The structure of (Z)-7e was confirmed by X-ray analysis (Figure S1). 


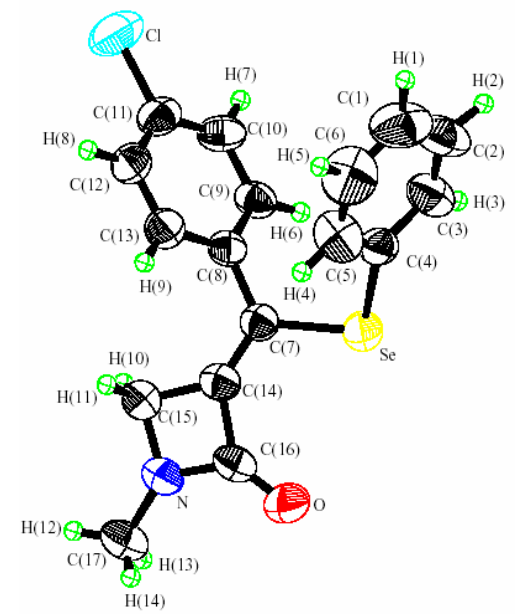

Figure S1. ORTEP Drawing of (Z)-7e

\section{(Z)-1-Butyl-3-phenylselenomethylenepiperidin-2-one (9a).}

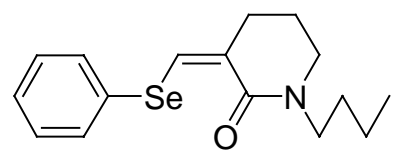

Into a 3-mL flask equipped with a reflux condenser were placed carbamoselenoate 8a $(0.4$ $\mathrm{mmol})$, toluene $(0.5 \mathrm{~mL})$, and $\mathrm{Pd}\left(\mathrm{PPh}_{3}\right)_{4}(0.020 \mathrm{mmol})$ at room temperature under $\mathrm{Ar}$ and the solution turned immediately red. After the mixture was refluxed for $1 \mathrm{~h}$, toluene was removed in vacuo. The crude product was purified by preparative TLC ( $n$-hexane/Et $2 \mathrm{O}=3 / 1$ ) to provide (Z)-1-butyl-3-phenylselenomethylenepiperidin-2-one (9a) in 75\% yield: colorless oil; ${ }^{1} \mathrm{H}$ NMR $\left(400 \mathrm{MHz}, \mathrm{CDCl}_{3}\right) \delta 0.94(\mathrm{t}, J=7.3 \mathrm{~Hz}, 3 \mathrm{H}), 1.30-1.39$ (tq, $\left.J=7.3,7.6 \mathrm{~Hz}, 2 \mathrm{H}\right), 1.54-1.62$ (dt, $J$ $=7.8,7.3 \mathrm{~Hz}, 2 \mathrm{H}), 1.85(\mathrm{t}, J=5.7 \mathrm{~Hz}, 2 \mathrm{H}), 2.52-2.55(\mathrm{~m}, 2 \mathrm{H}), 3.34(\mathrm{t}, J=5.7 \mathrm{~Hz}, 2 \mathrm{H}), 3.42(\mathrm{t}, J$ $=7.6 \mathrm{~Hz}, 2 \mathrm{H}), 7.05(\mathrm{~s}, 1 \mathrm{H}), 7.28-7.62(\mathrm{~m}, 5 \mathrm{H}) ;{ }^{13} \mathrm{C} \mathrm{NMR}\left(100 \mathrm{MHz}, \mathrm{CDCl}_{3}\right) \delta$ 14.0, 20.3, 22.9, $29.3,31.2,47.4,48.2,125.1,127.2,128.7,132.9\left({ }^{2} J_{S e-C}=9.2 \mathrm{~Hz}\right), 134.9,137.3\left({ }^{1} J_{S e-C}=131.4 \mathrm{~Hz}\right)$, 164.5; NOE experiment: Irradiation of the allylic proton multiplet at $\delta 2.53$ resulted in $8.3 \%$ and $8.3 \%$ enhancements of the signals at $\delta 1.85$ (C-2 methylene triplet) and $\delta 7.05$ (vinyl singlet), respectively; $\mathrm{IR}(\mathrm{NaCl})$ 3330, 2930, 2512, 2102, $1634(\mathrm{C}=\mathrm{O}), 1580,1487,1348,1236,1198,828$, 
740, $695 \mathrm{~cm}^{-1}$; MS(EI), m/e (relative intensity, \%) $323\left(\mathrm{M}^{+}, 100\right), 294$ (12), 280 (40), 246 (54), 190 (25), 157 (58), 143 (19), 123 (53), 95 (66), 77 (28). Anal. Calcd for $\mathrm{C}_{16} \mathrm{H}_{21} \mathrm{NOSe}$ : C, 59.62; H, 6.57; N, 4.35. Found: C, 59.52; H, 6.51; N, 4.31.

\section{(Z)-1-Butyl-3-phenylselenomethyleneazepan-2-one (9b).}

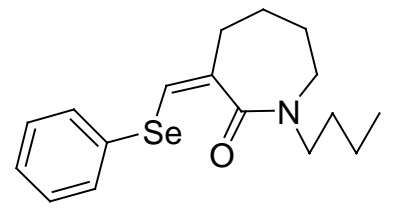

Into a 3-mL flask equipped with a reflux condenser were placed carbamoselenoate $\mathbf{8 b}(0.4$ mmol), toluene $(2 \mathrm{~mL})$, and $\mathrm{Pd}\left(\mathrm{PPh}_{3}\right)_{4}(0.020 \mathrm{mmol})$ at room temperature under Ar and the solution turned immediately red. After the mixture was refluxed for $12 \mathrm{~h}$, toluene was removed in vacuo. The crude product was purified by preparative TLC ( $n$-hexane/ $\mathrm{Et}_{2} \mathrm{O}=2 / 1$ ) to provide (Z)-1-butyl-3-phenylselenomethyleneazepan-2-one (9b) in $74 \%$ yield: colorless oil; ${ }^{1} \mathrm{H}$ NMR $\left(400 \mathrm{MHz}, \mathrm{CDCl}_{3}\right) \delta 0.95(\mathrm{t}, J=7.6 \mathrm{~Hz}, 3 \mathrm{H}), 1.33-1.76(\mathrm{~m}, 8 \mathrm{H}), 2.41-2.44(\mathrm{~m}, 2 \mathrm{H}), 3.35(\mathrm{t}, J=$

$6.1 \mathrm{~Hz}, 2 \mathrm{H}), 3.44(\mathrm{t}, J=7.6 \mathrm{~Hz}, 2 \mathrm{H}), 6.86(\mathrm{~s}, 1 \mathrm{H}), 7.27-7.60(\mathrm{~m}, 5 \mathrm{H}) ;{ }^{13} \mathrm{C} \mathrm{NMR}(100 \mathrm{MHz}$, $\left.\mathrm{CDCl}_{3}\right) \delta 14.1,20.3,27.2,27.3,30.3,33.0,48.2,48.3,127.1,128.9,130.7,132.5,132.8,136.1$, 164.5; NOE experiment: Irradiation of the allylic proton multiplet at $\delta 2.42$ resulted in $12.7 \%$ and $7.8 \%$ enhancements of the signals at $\delta 1.73$ (C-2 methylene multiplet) and $\delta 6.86$ (vinyl singlet), respectively; IR(NaCl) 2930, $1621(\mathrm{C}=\mathrm{O}), 1477,1423,1236,822,741,693 \mathrm{~cm}^{-1} ; \mathrm{MS}(\mathrm{EI}), \mathrm{m} / \mathrm{e}$ (relative intensity, \%) $337\left(\mathrm{M}^{+}, 100\right), 294$ (28), 256 (31), 206 (12), 180 (38), 157 (15), 138 (35), 126 (34), 77 (19). Anal. Calcd for $\mathrm{C}_{17} \mathrm{H}_{23}$ NOSe: C, 60.71; H, 6.89; N, 4.16. Found: C, 60.56; H, $6.93 ; \mathrm{N}, 4.21$. 


\section{1-Pent-4-ynyl-3-phenylselenomethyleneazetidin-2-one (11a).}

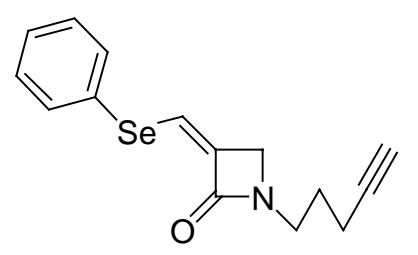

and

1-Prop-2-ynyl-3-phenylselenomethylenepiperidin-2-one (11b).

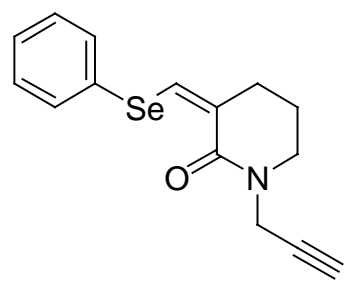

Into a 3-mL flask equipped with a reflux condenser were placed carbamoselenoate $\mathbf{1 0}(0.85$ mmol), toluene $(4 \mathrm{~mL})$, and $\mathrm{Pd}\left(\mathrm{PPh}_{3}\right)_{4}(0.085 \mathrm{mmol})$ at room temperature under Ar and the solution turned immediately red. After the mixture was refluxed for $1 \mathrm{~h}$, toluene was removed in vacuo. The crude product was purified by preparative TLC ( $n$-hexane/Et ${ }_{2} \mathrm{O}=2 / 1$ ) to provide colorless oil of 11a $(50 \%, E / Z=5 / 95)$ and white solid of $11 \mathbf{b}(10 \%, E / Z=11 / 89)$. The following spectra and analytical data were obtained from $E / Z$ mixtures.

11a: ${ }^{1} \mathrm{H}$ NMR $\left(400 \mathrm{MHz}, \mathrm{CDCl}_{3}\right) \delta 1.77-1.84(\mathrm{tt}, J=7.1,6.8 \mathrm{~Hz}, 3 \mathrm{H}), 2.00(\mathrm{t}, J=2.7 \mathrm{~Hz}, 1 \mathrm{H})$, 2.24-2.28 (dt, $J=2.7,7.1 \mathrm{~Hz}, 2 \mathrm{H}), 3.44(\mathrm{t}, J=6.8 \mathrm{~Hz}, 2 \mathrm{H}), 3.51(\mathrm{~s}, E(2 \mathrm{H})), 3.75(\mathrm{~s}, Z(2 \mathrm{H}))$, $6.73(\mathrm{~s}, Z(1 \mathrm{H})), 7.22(\mathrm{~s}, E(1 \mathrm{H})), 7.31-7.57(\mathrm{~m}, 5 \mathrm{H}),{ }^{13} \mathrm{C} \mathrm{NMR}\left(100 \mathrm{MHz}, \mathrm{CDCl}_{3}\right) \delta 16.1(E, Z)$, $26.5(E), 26.6(Z), 40.9(E), 41.0(Z), 48.4(E), 49.2(Z), 69.1,82.6,118.5,120.1\left({ }^{1} J_{S e-C}=122.2 \mathrm{~Hz}\right.$, (Z)), 127.6, 128.1, 129.0, 129.1, 129.9, $132.5\left({ }^{2} J_{S e-C}=11.1 \mathrm{~Hz},(Z)\right), 133.3,134.3,138.0,162.7$ (Z); NOE experiment: Irradiation of the allylic proton singlet at $\delta 3.75$ resulted in $3.9 \%$ enhancement of the signal at $\delta 6.73$ (vinyl singlet); IR(NaCl) 3293, 3055, 2948, $1732(\mathrm{C}=\mathrm{O})$, 1674, 1478, 1439, 1393, 1247, 1118, 1022, 826, 742, $692 \mathrm{~cm}^{-1}$; MS(EI), m/e (relative intensity, \%) 
$305\left(\mathrm{M}^{+}, 16\right), 224$ (76), 195 (27), 157 (23), 148 (100), 115 (41), 95 (14), 77 (29). Anal. Calcd for $\mathrm{C}_{15} \mathrm{H}_{15} \mathrm{NOSe}$ : C, 59.22; H, 4.97; N, 4.60. Found: C, 58.83; H, 4.84; N, 4.58.

11b: $\mathrm{mp} 78^{\circ} \mathrm{C} ;{ }^{1} \mathrm{H}$ NMR $\left(400 \mathrm{MHz}, \mathrm{CDCl}_{3}\right) \delta 1.89-1.95(\mathrm{tt}, J=6.1,5.9 \mathrm{~Hz}, 3 \mathrm{H}), 2.20(\mathrm{t}, J=2.4$ $\mathrm{Hz}, 1 \mathrm{H}), 2.55-2.58(\mathrm{t}, J=6.1 \mathrm{~Hz}, 2 \mathrm{H}), 3.34(\mathrm{t}, J=5.7 \mathrm{~Hz}, E(2 \mathrm{H})), 3.49(\mathrm{t}, J=5.9 \mathrm{~Hz}, Z(2 \mathrm{H}))$, $4.12(\mathrm{~d}, J=6.3 \mathrm{~Hz}, E(2 \mathrm{H})), 4.33(\mathrm{~d}, J=2.4 \mathrm{~Hz}, Z(2 \mathrm{H})), 7.15(\mathrm{~s}, Z(1 \mathrm{H})), 7.30-7.63(\mathrm{~m}, 5 \mathrm{H}) ;{ }^{13} \mathrm{C}$ NMR (100 MHz, $\left.\mathrm{CDCl}_{3}\right) \delta 22.2(E), 22.5(Z), 27.8(E), 31.0(Z), 35.7(Z), 36.1(E), 46.9(E), 47.1$ (Z), 71.5, 71.7, 77.0, 78.3, 124.5, 127.3, 127.6, 128.7, 129.0, 132.6, $132.9\left({ }^{2} J_{S e-C}=9.2 \mathrm{~Hz},(Z)\right)$, $134.4,139.1\left({ }^{1} J_{S e-C}=133.2 \mathrm{~Hz},(Z)\right), 164.3(Z)$; NOE experiment: Irradiation of the allylic proton triplet at $\delta 2.53$ resulted in $7.3 \%$ and $6.5 \%$ enhancements of the signals at $\delta 1.92$ (C-3 methylene quintet) and $\delta 7.15$ (vinyl singlet), respectively.; $\operatorname{IR}(\mathrm{KBr}) 3252,2943,1624(\mathrm{C}=\mathrm{O}), 1578,1486$, 1351, 1234, 1198, 947, 831, 741, $696 \mathrm{~cm}^{-1}$; MS(EI), m/e (relative intensity, \%) $305\left(\mathrm{M}^{+}, 100\right), 303$ (50), 225 (82), 157 (15), 148 (11), 129 (13), 115 (13), 77 (20). $\mathrm{C}_{15} \mathrm{H}_{15} \mathrm{NOSe}$ C, 59.22; H, 4.97; N, 4.60. Found: C, 59.09; H, 4.96; N, 4.61.

\section{(Z)-1-Methyl-3-phenylthiomethyleneazetidin-2-one (13, eq. 5).}

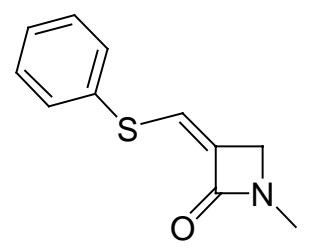

Into a 3-mL flask equipped with a reflux condenser were placed carbamothioate $12(0.4 \mathrm{mmol})$, toluene $(0.5 \mathrm{~mL})$, and $\mathrm{Pd}\left(\mathrm{PPh}_{3}\right)_{4}(0.020 \mathrm{mmol})$ at room temperature under $\mathrm{Ar}$ and the solution turned immediately red. After the mixture was refluxed for $1 \mathrm{~h}$, toluene was removed in vacuo. The crude product was purified by silica gel clolumn chromathography $\left(n\right.$-hexane/ $\left.\mathrm{Et}_{2} \mathrm{O}=3 / 1\right)$ to provide (Z)-1-methyl-3-phenylthiomethyleneazetidin-2-one (13) in 72\% yield: pale yellow solid; mp $128{ }^{\circ} \mathrm{C} ;{ }^{1} \mathrm{H}$ NMR $\left(400 \mathrm{MHz}, \mathrm{CDCl}_{3}\right) \delta 2.98(\mathrm{~s}, 3 \mathrm{H}), 3.73(\mathrm{~s}, 2 \mathrm{H}), 6.40(\mathrm{~s}, 1 \mathrm{H})$, 7.27-7.43 (m, 
$5 \mathrm{H}) ;{ }^{13} \mathrm{C} \mathrm{NMR}\left(100 \mathrm{MHz}, \mathrm{CDCl}_{3}\right) \delta 28.8,49.6,122.7,127.2,128.9,129.7,133.3,134.5,162.2$;

NOE experiment: Irradiation of the allylic proton singlet at $\delta 3.73$ resulted in $1.6 \%$ enhancement of the signal at $\delta 6.40$ (vinyl singlet); $\operatorname{IR}(\mathrm{KBr}) 3037,2948,1726(\mathrm{C}=\mathrm{O}), 1582,1480,1384,1253$, 1193, 1088, 1031, 901, 843, 792, 749, $692 \mathrm{~cm}^{-1}$; MS(EI), m/e (relative intensity, \%) $205\left(\mathrm{M}^{+}, 73\right)$, 147 (100), 134 (28), 109 (10), 96 (4), 77(5). Anal. Calcd for $\mathrm{C}_{11} \mathrm{H}_{11} \mathrm{NOS}$ : C, 64.36; H, 5.40; N, 6.82. Found: C, 64.08; H, 5.31; N, 6.80 .

\section{2,2-Dimethyl-4-phenylselenomethylenecyclobutanone (15).}

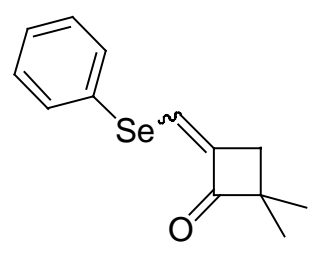

Into a 3-mL flask equipped with a reflux condenser were placed selenol ester $14(0.96 \mathrm{mmol})$, toluene $(1.25 \mathrm{~mL})$, and $\mathrm{Pd}\left(\mathrm{PPh}_{3}\right)_{4}(0.048 \mathrm{mmol})$ at room temperature under $\mathrm{Ar}$ and the solution turned immediately red. The mixture was refluxed for $1 \mathrm{~h}$ and toluene was removed in vacuo. After the NMR yield and the $E / Z$ ratio were determined by ${ }^{1} \mathrm{H}$ NMR $(73 \%, E / Z=74 / 26)$, the crude product was purified by twice preparative TLC ( $n$-hexane/Et ${ }_{2} \mathrm{O}=8 / 1$ and $n$-hexane/ $/ \mathrm{Et}_{2} \mathrm{O}=20 / 1$, respectively) to provide pure (E)- and (Z)-15.

(Z)-15: yellow solid: mp $39^{\circ} \mathrm{C} ;{ }^{1} \mathrm{H}$ NMR (400 MHz, $\left.\mathrm{CDCl}_{3}\right) \delta 1.25(\mathrm{~s}, 6 \mathrm{H}), 2.42(\mathrm{~d}, J=2.0 \mathrm{~Hz}, 2$ $\mathrm{H}), 7.00(\mathrm{t}, J=2.0 \mathrm{~Hz}, 1 \mathrm{H}), 7.33-7.36(\mathrm{~m}, 3 \mathrm{H}), 7.56-7.59(\mathrm{~m}, 2 \mathrm{H}) ;{ }^{13} \mathrm{C} \mathrm{NMR}\left(100 \mathrm{MHz}, \mathrm{CDCl}_{3}\right)$ $\delta 22.6,39.9,56.6,120.9,128.0,129.3,130.5,133.3\left({ }^{2} J_{S e-C}=11.1 \mathrm{~Hz}\right), 139.2,203.7 ; \quad$ NOE experiment: Irradiation of the allylic proton doublet at $\delta 2.42$ resulted in $2.3 \%$ enhancement of the signal at $\delta 7.00$ (vinyl singlet); $\mathrm{IR}(\mathrm{KBr})$ 2924, $1729(\mathrm{C}=\mathrm{O}), 1602,1478,1439,1275,1058,999$, 742, 692, $465 \mathrm{~cm}^{-1}$; MS(EI), m/e (relative intensity, \%) $266\left(\mathrm{M}^{+}, 37\right), 238$ (29), 182 (83), 180 (41), 157 (23), 109 (59), 102 (42), 81 (100), 77 (44). HRMS calcd for $\mathrm{C}_{13} \mathrm{H}_{14} \mathrm{OSe}$ 266.0210. Found 
266.0204 .

(E)-15: pale yellow solid: $\mathrm{mp} 67{ }^{\circ} \mathrm{C} ;{ }^{1} \mathrm{H}$ NMR $\left(400 \mathrm{MHz}, \mathrm{CDCl}_{3}\right) \delta 1.24(\mathrm{~s}, 6 \mathrm{H}), 2.24(\mathrm{~d}, J=2.9$ $\mathrm{Hz}, 2 \mathrm{H}), 7.33-7.36(\mathrm{~m}, 3 \mathrm{H}), 7.55-7.57(\mathrm{~m}, 2 \mathrm{H}), 7.72(\mathrm{t}, J=2.9 \mathrm{~Hz}, 1 \mathrm{H}) ;{ }^{13} \mathrm{C} \mathrm{NMR}(100 \mathrm{MHz}$, $\left.\mathrm{CDCl}_{3}\right) \delta 22.6,38.6,56.7,128.0,128.3,129.3,130.5,133.3\left({ }^{2} J_{S e-C}=10.6 \mathrm{~Hz}\right), 143.0,200.8$; $\mathrm{IR}(\mathrm{KBr})$ 2954, $1733(\mathrm{C}=\mathrm{O}), 1609,1466,1438,1269,1183,1089,1020,832,743,688,466 \mathrm{~cm}^{-1}$; MS(EI), m/e (relative intensity, \%) $266\left(\mathrm{M}^{+}, 24\right), 238$ (33), 182 (47), 180 (23), 157 (18), 109 (20), 102 (25), 81 (100), 77 (33). HRMS calcd for $\mathrm{C}_{13} \mathrm{H}_{14} \mathrm{OSe}$ : 266.0210. Found 266.0219. The structure of $(E)$-15 was confirmed by X-ray analysis (Figure S2).

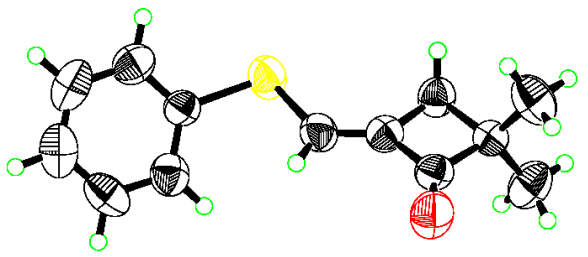

Figure S2. ORTEP Drawing of $(E)-15$

\section{References and Notes}

S1) Malpass, J. R.; Hemmings, D. A.; Wallis, A. L.; Fletcher, S. R.; Patel, S. J. Chem. Soc., Perkin Trans. 1, 2001, 1044.

S2) Eckert, H.; Forster, B. Angew. Chem., Int. Ed. Engl. 1987, 26, 894.

S3) 2,2-Dimethylpent-4-ynoyl chloride was prepared from the corresponding ethyl ester by hydrolysis with $\mathrm{KOH} / \mathrm{EtOH}$ and subsequent chlorination with $(\mathrm{COCl})_{2}$ : Magnus, P.; Slater, M. J.; Principe, L. M. J. Org. Chem. 1989, 54, 5148. 